Published in final edited form as:

Sex Transm Dis. 2020 March ; 47(3): 192-196. doi:10.1097/OLQ.0000000000001121.

\title{
An Assessment of Risk Factors for HSV-2 Infection in Malawian Women Using Two Classifications for the HerpeSelect 2 Test
}

\author{
Payal Chakraborty, MS ${ }^{1}$, Alison H. Norris, MD, PhD ${ }^{1,2}$, Sarah Huber-Krum, MPA, MSW, \\ $\mathrm{PhD}^{3}$, Sarah Garver, $\mathrm{PhD}^{4}$, Robert B. Hood, $\mathrm{MPH}^{1}$, Venson Banda ${ }^{5}$, Allahna Esber, MSPH, \\ $\mathrm{PhD}^{6}$, Patricia Carr Reese, MPH, MD ${ }^{7}$, Robert Krysiak, MS $^{8}$, Abigail Norris Turner, MSPH, \\ $\mathrm{PhD}^{2}$ \\ 1.The Ohio State University, Division of Epidemiology, College of Public Health, Columbus, $\mathrm{OH}$ \\ 43210, U.S. \\ 2.The Ohio State University, Division of Infectious Diseases, College of Medicine, Columbus, $\mathrm{OH}$ \\ 43210, U.S. \\ 3.Harvard T.H. Chan School of Public Health, Department of Global Health and Population, \\ Boston, MA 02120, U.S. \\ 4. University of Chicago, Department of Sociology, Chicago, IL 60637, U.S. \\ 5.Child Legacy International, Lilongwe, Malawi \\ 6. Henry M. Jackson Foundation for the Advancement of Military Medicine, Bethesda, MD, USA \\ 7.Lancaster General Hospital, Family Medicine Residency, Lancaster, PA, USA \\ 8.UNC Project, Lilongwe, Malawi
}

\section{Abstract}

Background: The HerpeSelect 2 ELISA IgG Test for herpes simplex virus type 2 (HSV-2) infection is widely used, convenient, and inexpensive. However, it has been shown to have lower specificity among populations in Sub-Saharan Africa compared to HSV-2 tests regarded as gold standards.

Methods: In 2016, we collected blood and survey data from 248 women participating in a community-based cohort study in rural Malawi (the Umoyo wa Thanzi (UTHA) project). Using multinomial logistic regression accounting for village-level clustering, we examined unadjusted associations between select demographic and sexual risk factors and HSV-2 serostatus. Because increasing the index value cutpoint for a positive result improves specificity, we coded HSV-2 serostatus in two ways: the manufacturer's recommended cutpoints $(<0.9=$ =negative, $0.9-$ $1.1=$ indeterminate, $>1.1=$ positive), and modified cutpoints with improved specificity $(<0.9=$ negative, $0.9-3.5=$ indeterminate, $>3.5=$ positive $)$. We aimed to investigate whether associations between select risk factors and HSV-2 serostatus varied under the two approaches.

Results: The prevalence of HSV-2 in this sample was $67 \%$ under the manufacturer's cutpoint and $22 \%$ under the modified cutpoint. Under both cutpoints, age, household size, number of marriages, 
and number of pregnancies were associated with HSV-2 positive serostatus. Using modified cutpoints, current bacterial vaginosis (odds ratio [OR]: 3.17 ; 95\% confidence interval [CI]: $1.35-$ 7.47), partner concurrency (OR: 4.88; 95\% CI: 2.54-9.37) and unsure about partner concurrency (OR: 1.91; 95\% CI: 1.08-3.38) were associated with HSV-2 seropositivity. In general, associations were attenuated under the recommended cutpoints compared to the modified cutpoints.

Conclusion: HSV-2-focused interventions informed by identifying individuals likely to have or acquire HSV-2 must be aware that different target populations may emerge depending on which cutpoints are adopted.

\section{Summary}

In a study of reproductive-aged women in Malawi, we found that correlates of HSV-2 positivity, diagnosed by the HerpeSelect 2 ELISA IgG test, changed when increasing the threshold for positivity.

\section{Keywords}

HSV-2; Malawi; ELISA

\section{Introduction}

Herpes simplex virus type 2 (HSV-2) is one of the most common sexually transmitted microbes, and HSV-2 infection cannot be cured. ${ }^{1}$ The prevalence of HSV-2 is higher in SubSaharan Africa than in other parts of the world. ${ }^{1-4} \mathrm{HSV}-2$ is a common cause of genital herpes, and while many people with HSV-2 infection are asymptomatic, in some individuals HSV-2 is associated with painful and recurrent lesions. Once tested and diagnosed, treatment can be given when symptoms arise. Furthermore, HSV-2 has a synergistic relationship with HIV. HSV-2 seroprevalence is an important population-level parameter in mathematical models of HIV transmission. For these reasons, low-cost, easy-to-administer tests to detect HSV-2 are important public health tools.

The HerpeSelect 2 ELISA IgG Test (Focus Technologies, Cypress, California) and Kalon HSV-2 gG2 ELISA (Kalon Biologicals Ltd, Aldershot, UK) are commonly used to detect HSV-2 infection, in part because they are inexpensive and convenient to use. However, these tests have demonstrated poor specificity in some regions of Sub-Saharan Africa. ${ }^{5-12}$ Low specificity has been attributed to cross-reactivity of the assay with other HSV types or unidentified antibodies to other infectious agents. In comparative studies done in SubSaharan Africa, the HerpeSelect 2 test has higher sensitivity and lower specificity than the Kalon test. ${ }^{5,7,11,13}$ One approach to improve test specificity while maintaining acceptable sensitivity is to increase the index value cutpoint for positivity for the HerpeSelect 2 test from 1.1 (the manufacturer's recommended cutpoint) to $2.1,2.2$, or 3.5. ${ }^{10,12}$ This allows use of the higher sensitivity for the HerpeSelect 2 test, relative to the Kalon test, in addition to improved specificity achieved by increasing the cutpoint for positivity.

Most studies that have evaluated performance of the HerpeSelect 2 test have evaluated its sensitivity and specificity using a gold standard diagnostic, such as Western Blot—regarded as the most accurate test ${ }^{14}$-or a monoclonal antibody-blocking enzyme immunoassay. ${ }^{12}$ 
However, no studies, to our knowledge, have examined whether the direction and strength of association between HSV-2 serostatus and specific demographic and sexual risk factorsincluding those identified in existing literature, such as age, household size, education, income, marital status, partner concurrency, frequency of sex, number of lifetime sexual partners, gravidity, and bacterial vaginosis ${ }^{1,15-17}$ — change when the index value cutpoint for positivity is increased to improve the specificity of the test. The purpose of the current analysis, conducted using data from reproductive-aged women in rural Malawi, was to investigate whether these associations changed when the diagnostic threshold was changed from the manufacturer's recommended cutpoints to a modified set of cutpoints.

\section{Methods}

\section{Study design and population}

Participants were part of the second wave of data collection for Umoyo wa Thanzi (UTHA), a community-based cohort study of sexual and reproductive health decision making in rural Lilongwe District, Malawi. To recruit the baseline wave (July 2014 to February 2015), we used stratified cluster sampling to select 11 clusters from the full sampling frame of 43 clusters within the catchment area of a community hospital. Clusters were chosen so that each cluster had 40 to 252 households. All women 15-39 years of age in the sampled villages were eligible to participate. In 2016, we randomly sampled a subset of seven clusters from those included in the baseline sample to re-interview 309 women into the second wave of data collection (Hood RB, Turner AN, Huber-Krum S, et al. \& Norris AH, Fokong K, Tompkins M, et al.). Out of these women, 248 and 210 women provided biological samples for HSV-2 infection and bacterial vaginosis, respectively. The research team visited the homes of participants in selected villages to tell them about the second wave of data collection. Those who agreed were interviewed and provided biological samples for subsequent testing.

\section{Informed consent}

During the baseline wave, written consent (or assent, for participants aged 15-17 years) was obtained for participation in the UTHA cohort prior to the interview. Participants were consented individually and in private, away from other members of the household. In the second wave, sampled participants from the baseline wave were approached for participation and were interviewed if they agreed to participate. All biological testing was optional, and participants were able to remain in the cohort even if they did not agree to provide biological samples.

\section{Participant procedures}

All participants were interviewed by trained data collectors in Chichewa. Participant responses were recorded using the Magpi electronic data capture system (Magpi, Washington, DC) on tablets. Data were uploaded into a cloud-based database at the end of each day.

For biological testing, we collected 10-20 drops of whole blood via finger prick using a sterile lance to create a dried blood spot. Dried blood spots were stored in a cool and dry 
environment until testing. All consenting women self-collected a vaginal swab, which was immediately rolled on a glass slide and allowed to air dry. HIV infection status was assessed using government provided rapid tests (Determine or Unigold).

\section{Lab procedures}

Dried blood spot samples were processed prior to HSV-2 testing according to the manufacturer's instructions. We diagnosed HSV-2 using the HerpeSelect 2 ELISA IgG test (Focus Diagnostics, Cypress, CA). We calculated sample index values by dividing specimen optical density values by the mean of the cut-off calibrator absorbance values per the manufacturer's instructions. Dried vaginal smears were Gram stained according to standard procedures and evaluated for bacterial vaginosis using Nugent scoring.

\section{HSV-2 serostatus classifications}

We examined HSV-2 serostatus using two classifications. First, per the manufacturer's instructions, index values less than 0.9 were considered negative, $0.9-1.1$ were indeterminate, and greater than 1.1 were considered positive for HSV-2. Samples that tested indeterminate were retested two additional times. If both repeat tests were positive (index value $>1.1$ ), we classified the sample as positive. If both repeat tests were negative (index value $<0.9$ ), we classified the sample as negative. If one of the repeat tests was positive and one was negative, or either was again indeterminate, the sample remained classified as indeterminate. Second, we used a modified set of cutpoints, in which index values less than 0.9 were considered negative, $0.9-3.5$ were indeterminate, and greater than 3.5 were considered HSV-2 seropositive. The cutoff of 3.5 was chosen because it has been commonly suggested for populations in Sub-Saharan Africa. ${ }^{10}$ Again, if the first test was indeterminate (i.e., index value between 0.9 and 3.5), we repeated the test two additional times to confirm the result.

\section{Statistical analysis}

All analyses were conducted using Stata 15 (StataCorp, College Station, TX). We reported descriptive statistics as frequencies and percentages for categorical variables, and means and standard deviations for continuous variables. Demographic and sexual factors of interest included age, number of people living in the household, income, marital status, concurrent partners, number of lifetime sexual partners, number of marriages, frequency of sex, bacterial vaginosis, and number of pregnancies. We used multinomial logistic regression to assess bivariate associations between each demographic and sexual risk factor and HSV-2, separately for the two classifications. Each model produced two odds ratios, one comparing positive to negative HSV-2 serostatus and one comparing indeterminate to negative serostatus. All analyses accounted for clustering at the village level using robust standard errors.

\section{Ethical approval}

The study was approved by the Ohio State University Institutional Review Board and the University of Malawi College of Medicine Research Ethics Committee. 


\section{Results}

\section{Participant characteristics}

Of the 309 women who were interviewed, 248 women provided blood for HSV-2 testing and thus were included in the analysis. The average age was 26 years (standard deviation [SD] 7$)$, and most of the participants ( $81 \%, \mathrm{~N}=196)$ were married (Table 1). Approximately a quarter $(23.6 \%, \mathrm{~N}=46)$ of women tested positive for bacterial vaginosis. A third (33.2\%, $\mathrm{N}=71$ ) of women reported that their partners were in a concurrent relationship and $33.2 \%$ $(\mathrm{N}=71)$ of women reported not knowing if their partners were in a concurrent relationship. The average household size was 4.7 people $(\mathrm{SD}=1.6)$, and the average number of pregnancies was $3.7(\mathrm{SD}=2.1)$. Under the manufacturer's cutpoints, 67\% $(\mathrm{N}=166)$ tested positive for HSV-2 infection, while $22 \%(\mathrm{~N}=54)$ tested positive using the modified cutpoints (Figure 1).

\section{Correlates of positive vs. negative HSV-2 serostatus, by classification threshold}

Older age, larger household size, higher number of marriages, and higher number of pregnancies were associated with positive vs. negative HSV-2 serostatus under both thresholds (Table 2). No variables were only associated with positive vs. negative HSV-2 serostatus using the manufacturer's cutpoints, but three variables were associated with positive HSV-2 serostatus using only the modified cutpoints, including having bacterial vaginosis (OR: $3.17 ; 95 \% \mathrm{CI}: 1.35,7.47$ ), knowing that the partner is in a concurrent relationship (OR: $4.88 ; 95 \%$ CI: 2.54, 9.37), and not knowing if the partner is in a concurrent relationship (OR: 1.91; 95\% CI: 1.08, 3.38), as compared to negative HSV-2 serostatus.

In general, when comparing positive to negative HSV-2 serostatus, the measures of effect were attenuated (e.g., closer to the null of OR 1.0) when using the manufacturer's less specific cutpoints compared to the more specific modified cutpoints (Table 2). For marital status and having a concurrent partner, the directions of the association switched based on the cutpoints used.

\section{Correlates of indeterminate vs. negative HSV-2 serostatus, by classification threshold}

No variable was significantly associated with indeterminate HSV-2 serostatus, compared to negative HSV-2 serostatus, using the manufacturer's cutpoints. Household size was the only variable significantly associated with indeterminate HSV-2 serostatus using the modified cutpoints (OR: 1.36; 95\% CI: 1.08, 1.70).

\section{Discussion}

Among this population of women in rural Malawi, changing the cutpoint for positivity for the HerpeSelect $2 \mathrm{HSV}-2$ test led to different conclusions regarding associations with previously identified demographic and sexual risk factors for HSV-2. Specifically, we generally observed strengthened measures of effect for the modified cutpoints compared to the manufacturer's cutpoints. 
In this sample, the prevalence of HSV-2 was $22 \%$ under the modified cutpoint. Other studies conducted in Malawi used the Kalon test to detect HSV-2 infection. An HSV-2 age-adjusted prevalence from 2002 to 2005 of $45.4 \%$ was reported in Karonga district. ${ }^{18}$ In another study conducted in Karonga District among women aged 15-30 years, the prevalence of HSV-2 was $25.5 \% .{ }^{19}$ In a sample of younger women (17-20 years old) from Machinga and Balaka districts, rural districts in Southern Malawi, 15\% of the sample tested positive for HSV-2 infection. ${ }^{20}$ The observed variations in prevalence may be due to geographical differences, or the time periods in which the studies were conducted.

Increasing the threshold for positivity, while increasing the specificity of the diagnostic test, necessarily changes the classification for some samples from positive to indeterminate. The goal of increasing the threshold was to reduce potential misclassification introduced by the manufacturer's recommended cutpoints. We posited that this misclassification could be differential with regard to the factors we examined. For example, older participants, or those with more marriages, may be more likely to have cross-reactive antibodies through exposure to higher numbers of sexual partners. Ultimately however, the findings align with conventional expectations in settings of non-differential misclassification: the resulting measures of effect under the manufacturer's cutpoints are closer to the null than those observed using the modified cutpoints.

We also found that bacterial vaginosis status and partner concurrency were significantly associated with HSV-2 serostatus using the modified cutpoints but not the manufacturer's cutpoints. Previous research has suggested that HSV-2 is a risk factor for bacterial vaginosis. ${ }^{16}$ Furthermore, partner concurrency is strongly linked to HSV-2 status, and HSV-2 status is often used as an indicator of higher-risk sexual practices. ${ }^{21}$ From a public health perspective, these well-established correlates of HSV-2 infection status may not have been identified in the study population if the manufacturer's cutpoints had been used.

Although the HerpeSelect 2 test is widely used, the test's low specificity in certain populations poses a challenge for researchers and public health practitioners. While researchers have recommended increasing the cutpoint for positivity to obtain more optimal levels of specificity, ${ }^{10,12}$ specificity values may vary based on the location studied. ${ }^{5-12}$ Indeed, there is little official guidance on what cutpoints (and even what tests) should be used to measure HSV-2 in populations in whom the specificity of the HerpeSelect 2 has been shown to be poor. The first studies that identified the low specificity of the HerpeSelect 2 test in certain populations were conducted in the early 2000s. Yet in 2019, the recommended cutoff for positivity remains an index value of 1.1 , which is based on validation samples from North American and European populations. The Western Blot, which is regarded as the most accurate test for diagnosis of HSV-2 infection, is expensive and infeasible to use for routine testing, especially in low-resource settings. Thus, despite the documented concerns, inexpensive and convenient tests such as the HerpeSelect 2 test continue to be used for routine clinical and prevention efforts.

Another consideration in deciding whether to use modified cutpoints for the HerpeSelect 2 test is the tradeoff between the high number of potentially false positives that result from the manufacturer's cutpoints, versus the high number of indeterminate results using the 
modified cutpoints. (In the present study, increasing the cutpoint for positivity widened the indeterminate range, and almost half of the sample subsequently fell in this category). Both of these scenarios can be problematic. False positive results may be traumatic for patients and may lead to expensive confirmatory testing or unnecessary prophylaxis to prevent onward sexual transmission. However, the clinical recommendations following an indeterminate HSV-2 result are also unclear, because it is not known if the patient is in an early stage of seroconversion or whether the result is a consequence of cross-reaction with other infections or another scenario leading to the indeterminate result. Furthermore, increasing the cutoff may lead truly HSV-2-positive people to be classified as indeterminate. 13

Because HSV-2 infection is subclinical in most people, experience of symptoms cannot be relied on to detect infection; ${ }^{14}$ cost-effective and accurate tests are important at both an individual- and population-level. At the individual-level, HSV-2 infection can lead to substantial morbidity, as genital herpes can cause painful lesions. ${ }^{14}$ Furthermore, HSV-2 infection is lifelong, and undiagnosed HSV-2 infection can be transmitted to sexual partners. HSV-2 infection status is important to know for people who are pregnant, or thinking of becoming pregnant; neonatal transmission of herpes during birth can cause severe neurological impairment or death to the neonate. ${ }^{4} \mathrm{HSV}-2$ can also increase the risk of acquiring HIV infection, as well as increase the clinical progression and transmission of HIV. $1,14,22$

Public health practitioners and epidemiologists also remain keenly interested in HSV-2 prevalence at the population level. The community prevalence of HSV-2 is used in mathematical models to predict HIV transmission through various populations. ${ }^{23}$ Ministries of Health and other government agencies may use these figures to prioritize interventions to reduce the burden of HSV-2. Thus, accurate, cost-effective, and routine testing for HSV-2 is important to identify the burden of HSV-2 in populations, overall and in key risk groups.

One limitation of this study is that the sample size was small, making multivariable adjustment infeasible. We also did not conduct additional testing, and thus, we could not determine participants' true HSV-2 status. As a result, we could not quantify how many participants were misclassified under the two sets of cutpoints. Also, the number of women who tested indeterminate using the manufacturer's cutpoints was particularly low, as expected due to the narrow index value range, but comparisons for this category were likely underpowered. Furthermore, although the drawn sample was representative of the clinic catchment area, because some people (approximately 20\%) declined to participate in the biological testing portion of the study, the final sample may not have been representative of the rural Malawian setting.

The present study is the first to our knowledge to compare associations between known risk factors of HSV-2 infection and HSV-2 positivity (as diagnosed by the HerpeSelect 2 test, or any test based on recombinant gG-2), according to different cutpoints for positivity. Previous studies have compared the HerpeSelect 2 test with the Western Blot; ${ }^{12}$ this type of investigation is what first identified the low specificity of the HerpeSelect 2 test at the manufacturer's cutpoint for some populations. Although researchers have suggested that 
more optimal specificity levels can be achieved for the HerpeSelect 2 test, little official guidance exists. For the US population, the 2015 CDC STD Treatment Guidelines recommend performing a confirmatory test if the HerpeSelect index value is between 1.1 and 3.5. Still, it is unclear if the chosen set of cutpoints would affect the robustness of the associations with previously identified HSV-2 risk factors. The findings from the present study suggest that associations may change based on the adopted cutpoints, and that there is a need for the development of guidelines regarding which cutpoints to use for different populations in order to aid HSV-2 research and clinical and public health practice.

\title{
Funding Support:
}

Support for this project was provided by the Ohio State University Institute for Population Research through a grant from the Eunice Kennedy Shriver National Institute for Child Health and Human Development of the National Institutes of Health [P2CHD058484], the OSU Public Health Preparedness for Infectious Diseases program, National Center for Advancing Translational Sciences [KL2TR002734], and the Bill and Melinda Gates Foundation.

The content is solely the responsibility of the authors and does not necessarily represent the official views of the National Center For Advancing Translational Sciences, the National Institutes of Health, or the Bill and Melinda Gates Foundation.

\author{
Abbreviations \\ HSV-2 \\ Herpes Simplex Virus Type 2 \\ UTHA Umoyo wa Thanzi \\ OR Odds Ratio \\ CI Confidence Interval \\ SD Standard Deviation
}

\section{References}

1. Looker KJ, Magaret AS, Turner KME, et al. Global estimates of prevalent and incident herpes simplex virus type 2 infections in 2012. PLoS One 2015; 10(1):e114989. [PubMed: 25608026]

2. Smith JS, Robinson NJ. Age-specific prevalence of infection with herpes simplex virus types 2 and 1: a global review. J Infect Dis 2002; 186 Suppl 1:S3-28. [PubMed: 12353183]

3. Weiss HA, Buve A, Robinson NJ, et al. The epidemiology of HSV-2 infection and its association with HIV infection in four urban African populations. AIDS 2001; 15 Suppl 4:S97-108. [PubMed: 11686471]

4. Looker KJ, Garnett GP, Schmid GP. An estimate of the global prevalence and incidence of herpes simplex virus type 2 infection. Bull World Health Organ 2008; 86(10):805-812, A. [PubMed: 18949218]

5. van Dyck E, Buve A, Weiss HA, et al. Performance of commercially available enzyme immunoassays for detection of antibodies against herpes simplex virus type 2 in African populations. J Clin Microbiol 2004; 42(7):2961-2965. [PubMed: 15243045]

6. Ashley-Morrow R, Nollkamper J, Robinson NJ, et al. Performance of Focus ELISA tests for herpes simplex virus type 1 (HSV-1) and HSV-2 antibodies among women in ten diverse geographical locations. Clin Microbiol Infect 2004; 10(6):530-536. [PubMed: 15191381]

7. Ng'ayo MO, Friedrich D, Holmes KK, et al. Performance of HSV-2 type specific serological tests in men in Kenya. J Virol Methods 2010; 163(2):276-281. [PubMed: 19854222] 
8. Laeyendecker O, Henson C, Gray RH, et al. Performance of a commercial, type-specific enzymelinked immunosorbent assay for detection of herpes simplex virus type 2-specific antibodies in Ugandans. J Clin Microbiol 2004; 42(4):1794-1796. [PubMed: 15071053]

9. Smith JS, Bailey RC, Westreich DJ, et al. Herpes simplex virus type 2 antibody detection performance in Kisumu, Kenya, using the Herpeselect ELISA, Kalon ELISA, Western blot and inhibition testing. Sex Transm Infect 2009; 85(2):92-96. [PubMed: 18955387]

10. Mujugira A, Morrow RA, Celum C, et al. Performance of the Focus HerpeSelect-2 enzyme immunoassay for the detection of herpes simplex virus type 2 antibodies in seven African countries. Sex Transm Infect 2011; 87(3):238-241. [PubMed: 21307152]

11. Delany-Moretlwe S, Jentsch U, Weiss H, et al. Comparison of Focus HerpesSelect and Kalon HSV-2 gG2 ELISA serological assays to detect herpes simplex virus type 2 antibodies in a South African population. Sex Transm Infect 2010; 86(1):46-50. [PubMed: 19837726]

12. Biraro S, Mayaud P, Morrow RA, et al. Performance of commercial herpes simplex virus type-2 antibody tests using serum samples from Sub-Saharan Africa: a systematic review and metaanalysis. Sex Transm Dis 2011; 38(2):140-147. [PubMed: 20706175]

13. LeGoff J, Mayaud P, Gresenguet G, et al. Performance of HerpeSelect and Kalon assays in detection of antibodies to herpes simplex virus type 2. J Clin Microbiol 2008; 46(6):1914-1918. [PubMed: 18385443]

14. Corey L, Wald A. Genital Herpes. In: Holmes KK, Sparling PF, Stamm WE, Piot P, Wasserheit JN, Corey L, Cohen MS, Watts DH, eds. Sexually Transmitted Diseases. 4th ed. New York: The McGraw-Hill Companies; 2007:399-437.

15. Rajagopal S, Magaret A, Mugo N, et al. Incidence of herpes simplex virus type 2 infections in Africa: a systematic review. Open Forum Infect Dis 2014; 1(2):ofu043. [PubMed: 25734115]

16. Esber A, Vicetti Miguel RD, et al. Risk of Bacterial Vaginosis Among Women With Herpes Simplex Virus Type 2 Infection: A Systematic Review and Meta-analysis. J Infect Dis 2015; 212(1):8-17. [PubMed: 25589333]

17. Wald A Herpes simplex virus type 2 transmission: risk factors and virus shedding. Herpes 2004; 11 Suppl 3:130A-137A.

18. Glynn JR, Crampin AC, Ngwira BMM, et al. Herpes simplex virus type 2 trends in relation to the HIV epidemic in northern Malawi. Sex Transm Infect 2008; 84(5):356-360. [PubMed: 18524842]

19. Glynn JR, Kayuni N, Gondwe L, et al. Earlier menarche is associated with a higher prevalence of Herpes simplex type-2 (HSV-2) in young women in rural Malawi. Elife 2014; 3:e01604. [PubMed: 24473074]

20. Sudfeld CR, Hewett PC, Abuelezam NN, et al. Herpes simplex virus type 2 cross-sectional seroprevalence and the estimated rate of neonatal infections among a cohort of rural Malawian female adolescents. Sex Transm Infect 2013; 89(7):561-567. [PubMed: 23794069]

21. Cowan FM, Johnson AM, Ashley R, et al. Antibody to herpes simplex virus type 2 as serological marker of sexual lifestyle in populations. BMJ 1994; 309(6965):1325-1329. [PubMed: 7866079]

22. Freeman EE, Weiss HA, Glynn JR, et al. Herpes simplex virus 2 infection increases HIV acquisition in men and women: systematic review and meta-analysis of longitudinal studies. AIDS 2006; 20(1):73-83. [PubMed: 16327322]

23. Fleming DT, Wasserheit JN. From epidemiological synergy to public health policy and practice: the contribution of other sexually transmitted diseases to sexual transmission of HIV infection. Sex Transm Infect 1999; 75(1):3-17. [PubMed: 10448335] 


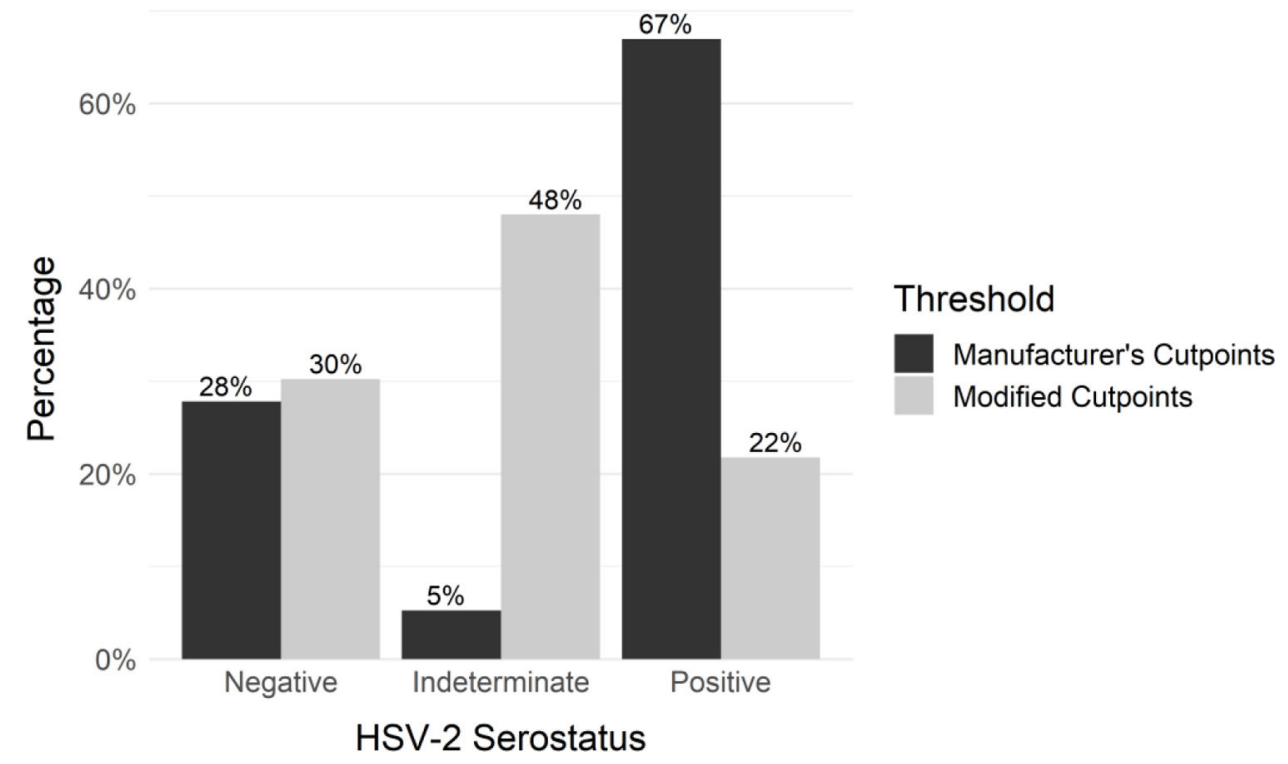

Figure 1.

HSV-2 serostatus by classification threshold $(\mathrm{N}=248)$

Manufacturer's recommended cutpoints: $<0.9=$ negative, $0.9-1.1=$ indeterminate,

$>1.1=$ positive; modified cutpoints (improved specificity): $<0.9=$ negative, $0.9-$

$3.5=$ indeterminate, $>3.5=$ positive 
Table 1.

Participant characteristics ( $\mathrm{N}=248$ ), Lilongwe District, Malawi, 2016

\begin{tabular}{|c|c|c|}
\hline Variable & $\mathbf{N}$ & $\%$ \\
\hline Bacterial Vaginosis & 46 & 23.6 \\
\hline \multicolumn{3}{|l|}{ HIV } \\
\hline Negative & 224 & 97.0 \\
\hline Positive & 7 & 3.0 \\
\hline \multicolumn{3}{|l|}{ Number of Marriages } \\
\hline $0-1$ & 170 & 70.0 \\
\hline 2 or More & 73 & 30.0 \\
\hline \multicolumn{3}{|l|}{ Education } \\
\hline$<2$ Years & 31 & 12.6 \\
\hline 2-4 Years & 94 & 38.2 \\
\hline 5-8 Years & 93 & 37.8 \\
\hline$>8$ Years & 28 & 11.4 \\
\hline \multicolumn{3}{|c|}{ Household Income (Malawian Kwacha) ${ }^{*}$} \\
\hline$<10,000$ & 89 & 46.4 \\
\hline $10,000-29,999$ & 51 & 26.6 \\
\hline$>30,000$ & 52 & 27.1 \\
\hline Married & 196 & 81.0 \\
\hline Concurrent Partner & 5 & 2.4 \\
\hline \multicolumn{3}{|c|}{ Partner in Concurrent Relationship } \\
\hline No & 72 & 33.6 \\
\hline Don't Know & 71 & 33.2 \\
\hline Yes & 71 & 33.2 \\
\hline \multicolumn{3}{|l|}{ Frequency of Sex } \\
\hline None or Infrequent & 50 & 26.9 \\
\hline Frequent & 136 & 73.1 \\
\hline \multicolumn{3}{|c|}{ Number of Lifetime Sexual Partners } \\
\hline 1 & 115 & 49.6 \\
\hline 2 & 71 & 30.6 \\
\hline 3 & 31 & 13.4 \\
\hline \multirow[t]{2}{*}{$\geq 4$} & 15 & 6.5 \\
\hline & Mean & SD \\
\hline Age & 25.8 & 6.9 \\
\hline Household Size & 4.7 & 1.6 \\
\hline Pregnancies & 3.7 & 2.1 \\
\hline
\end{tabular}

Sex Transm Dis. Author manuscript; available in PMC 2021 January 20. 
Table 2.

Unadjusted odds ratios (OR) and 95\% confidence intervals (95\% CI) between HSV-2 Serostatus and Select Risk Factors

\begin{tabular}{|c|c|c|c|c|c|c|c|c|}
\hline & \multicolumn{4}{|c|}{ Positive vs. Negative } & \multicolumn{4}{|c|}{ Indeterminate vs. Negative } \\
\hline & \multicolumn{2}{|c|}{ Manufacturer's Cutpoints } & \multicolumn{2}{|c|}{ Modified Cutpoints } & \multicolumn{2}{|c|}{ Manufacturer's Cutpoints } & \multicolumn{2}{|c|}{ Modified Cutpoints } \\
\hline & OR & $95 \% \mathrm{CI}$ & OR & $95 \% \mathrm{CI}$ & OR & $95 \% \mathrm{CI}$ & OR & $95 \% \mathrm{CI}$ \\
\hline Age (per 1-year increase) & 1.06 & $(1.03,1.10)$ & 1.14 & $(1.09,1.18)$ & 1.07 & $(0.98,1.17)$ & 1.04 & $(0.99,1.09)$ \\
\hline $\begin{array}{l}\text { Household Size (per 1-person } \\
\text { increase) }\end{array}$ & 1.42 & $(1.18,1.70)$ & 1.46 & $(1.23,1.72)$ & 1.26 & $(0.97,1.63)$ & 1.36 & $(1.08,1.70)$ \\
\hline \multicolumn{9}{|l|}{ Education (Ref: <2 years) } \\
\hline 2-4 Years & 0.17 & $(0.04,0.73)$ & 0.15 & $(0.03,0.69)$ & 0.19 & $(0.03,1.21)$ & 0.15 & $(0.04,0.57)$ \\
\hline$>5$ Years & 0.14 & $(0.03,0.66)$ & 0.08 & $(0.02,0.44)$ & 0.15 & $(0.02,1.09)$ & 0.17 & $(0.04,0.68)$ \\
\hline \multicolumn{9}{|l|}{$\begin{array}{l}\text { Household Income (Ref: } \\
<10,000)\end{array}$} \\
\hline $10,000-29,999$ & 0.79 & $(0.41,1.53)$ & 0.74 & $(0.31,1.81)$ & 1.53 & $(0.33,7.14)$ & 0.91 & $(0.52,1.59)$ \\
\hline$>30,000$ & 0.95 & $(0.33,2.78)$ & 0.82 & $(0.30,2.25)$ & 0.82 & $(0.10,7.06)$ & 0.91 & $(0.36,2.30)$ \\
\hline Married & 0.73 & $(0.35,1.53)$ & 1.70 & $(0.30,9.65)$ & 0.40 & $(0.08,1.95)$ & 0.53 & $(0.28,0.99)$ \\
\hline Concurrent Partner & 0.61 & $(0.18,2.00)$ & 1.32 & $(0.33,5.32)$ & --- & --- & 0.31 & $(0.08,1.16)$ \\
\hline \multicolumn{9}{|l|}{$\begin{array}{l}\text { Partner in Concurrent } \\
\text { Relationship (Ref: No) }\end{array}$} \\
\hline Don’t Know & 1.09 & $(0.62,1.90)$ & 1.91 & $(1.08,3.38)$ & 1.64 & $(0.23,11.85)$ & 1.19 & $(0.66,2.17)$ \\
\hline Yes & 1.94 & $(0.93,4.06)$ & 4.88 & $(2.54,9.37)$ & 4.29 & $(0.74,24.98)$ & 1.36 & $(0.62,2.95)$ \\
\hline \multicolumn{9}{|l|}{$\begin{array}{l}\text { Frequency of Sex (Ref: < Once } \\
\text { Per Week) }\end{array}$} \\
\hline > Once Per Week & 0.48 & $(0.21,1.08)$ & 0.50 & $(0.17,1.45)$ & 0.75 & $(0.16,3.57)$ & 0.51 & $(0.22,1.17)$ \\
\hline \multicolumn{9}{|l|}{$\begin{array}{l}\text { No. of Sexual Partners (Ref: } \\
\text { One) }\end{array}$} \\
\hline Two & 1.12 & $(0.67,1.90)$ & 2.08 & $(1.00,4.32)$ & 0.42 & $(0.12,1.47)$ & 0.78 & $(0.44,1.40)$ \\
\hline Three or More & 1.41 & $(0.84,2.37)$ & 2.05 & $(0.94,4.47)$ & 1.20 & $(0.39,3.68)$ & 0.94 & $(0.52,1.70)$ \\
\hline \multicolumn{9}{|l|}{ No. of Marriages (Ref: 0-1) } \\
\hline$>2$ & 2.03 & $(1.14,3.61)$ & 3.66 & $(1.84,7.27)$ & 0.31 & $(0.05,2.08)$ & 1.41 & $(0.63,3.18)$ \\
\hline Bacterial Vaginosis & 1.88 & $(0.89,3.97)$ & 3.17 & $(1.35,7.47)$ & 1.28 & $(0.25,6.49)$ & 1.79 & $(0.91,3.50)$ \\
\hline $\begin{array}{l}\text { Pregnancies (per 1-pregnancy } \\
\text { increase) }\end{array}$ & 1.26 & $(1.10,1.43)$ & 1.38 & $(1.21,1.58)$ & 1.19 & $(0.95,1.50)$ & 1.15 & $(1.00,1.33)$ \\
\hline
\end{tabular}

$\mathrm{OR}=$ odds ratio $\mathrm{CI}=$ confidence interval 